\title{
Treating aging: progress toward dietary restriction mimetics
} Filipe Cabreiro and David Gems*

\author{
Address: Institute of Healthy Ageing and Department of Genetics, Evolution \& Environment, University College London, Darwin Building, \\ Gower Street, London WC1E 6BT, UK \\ *Corresponding author: David Gems (david.gems@ucl.ac.uk) \\ FI000 Biology Reports 2010, 2:76 (doi:10.34I0/B2-76) \\ This is an open-access article distributed under the terms of the Creative Commons Attribution-Non Commercial License \\ (http://creativecommons.org/licenses/by-nc/3.0/legalcode), which permits unrestricted use, distribution, and reproduction in any medium, \\ provided the original work is properly cited. You may not use this work for commercial purposes. \\ The electronic version of this article is the complete one and can be found at: http://f1000.com/reports/b/2/76
}

\begin{abstract}
During the last decade, biogerontologists have labored to understand the biological basis of the aging process by studying the genes and signaling pathways that regulate it. But the last year has seen a breakthrough in a different direction: toward treatments that might slow aging by mimicking the effects of dietary restriction.
\end{abstract}

\section{Introduction and context}

The effects of biological aging make up a sizeable proportion of the sum of human suffering. Aging is the predominant risk factor for the many horrible illnesses of later life, including cardiovascular disease, cancer, and neurodegenerative conditions such as Alzheimer's disease. From a biological perspective, aging very much resembles a complex disease syndrome $[1,2]$. Because of this complexity, treatments for aging for have long seemed a forlorn hope. Moreover, the biological mechanisms of aging remain uncertain, although several promising hypotheses (e.g., that aging is caused by the accumulation of molecular damage) are currently under investigation [3]. Yet recent developments suggest that it might be feasible to treat aging even without fully understanding it.

In laboratory animals, it is possible to slow aging by using a variety of interventions - dietary, genetic, and pharmacological. For example, dietary restriction (DR), the measured reduction of food intake without starvation, can increase life span in organisms ranging from budding yeast (Saccharomyces cerevisiae) to dogs [4]. An important detail is that organisms under DR remain in a youthful state into late life and are resistant to agingassociated pathologies. This is consistent with the view of aging as a single, complex disease syndrome.

How does DR act to slow aging? One idea is that it affects nutrient-sensitive endocrine and intracellular signaling pathways that regulate aging. This is supported by studies of long-lived mutants (e.g., in the nematode worm Caenorhabditis elegans and the fruit fly Drosophila melanogaster). These studies have identified a network of nutrient- and stress-sensitive pathways that control aging. For example, mutations that reduce insulin/ insulin-like growth factor-1 signaling (IIS) can increase adult life spans in worms (as much as 10-fold [5]), flies, and mice [6].

Were these DR-responsive pathways druggable, then, in principle, one could induce a DR-like state with resistance to aging-related disease. Such DR mimetic drugs could be an efficient way to improve late-life health and well-being in the future [7]. Several nutrient-sensing pathways, including IIS and the $\mathrm{NAD}^{+}$-dependent histone deacetylases (sirtuins), have been postulated to mediate the effects of DR [4]. Initial reports suggested that DR-induced longevity in yeast, worms, and flies was attributable to sirtuin activation, although this was subsequently challenged [4]. Also pursued were sirtuinactivating DR mimetic drugs such as the plant-derived polyphenol resveratrol. However, initial observations of sirtuin activation by resveratrol seem attributable to experimental artefact [8].

Recent studies have focused increasingly on a different nutrient-sensing pathway, which includes target of rapamycin (TOR) kinase and ribosomal S6 kinase (S6K). 
This pathway regulates cell growth, ribosome biogenesis, and protein turnover. Evidence from yeast, worms, and flies suggests that this pathway mediates the effects of DR on life span $[4,6]$. The proximal mechanisms involved remain unclear, although a candidate effector in yeast is the nutrient-sensitive transcription factor Gcn4 [9]. These findings raise a number of questions: Does the TOR/S6K pathway influence aging and mediate the effects of DR in mammals? Could drugs that target this pathway act as DR mimetics? Could these work in humans? This last year has seen progress in providing answers to all of these questions.

\section{Major recent advances}

First, it was shown that mutation-induced loss of mouse S6K1 increases life span and resistance to age-related pathologies [10]. Also, the physiology and gene expression of S6K1 mutant mice resembled those of wild-type mice subjected to DR. This suggests that, as in lower organisms, TOR/S6K signaling mediates DR effects. If this view is correct, then pharmacological inhibition of this pathway should recapitulate the effects of DR. Consistent with this, it was recently shown that the TOR inhibitor rapamycin (sirolimus) increases life span in flies [11] and also in mice, in which it was administered from mid-life onwards [12]. This perhaps suggests broad efficacy of DR mimetics consumed in later life.

S6K1 mutant mice also showed elevated activation of the nutrition-sensitive enzyme AMPK (adenosine monophosphate-activated kinase) [10], hinting that AMPK activation might promote longevity under DR. In C. elegans, longevity induced by loss of S6K (rsks-1) proved to be AMPK-dependent [10], supporting this view. The biguanide drug metformin, widely used as a treatment for type 2 diabetes, activates AMPK, inhibits TOR [13], and can induce a DR-like mRNA profile in mice [14]. It can also increase life span in both worms [15] and short-lived mouse strains [16] but not in male rats [17]. However, aging seems to be more

Figure I. Dietary restriction (DR) mimetic studies from model organisms to humans

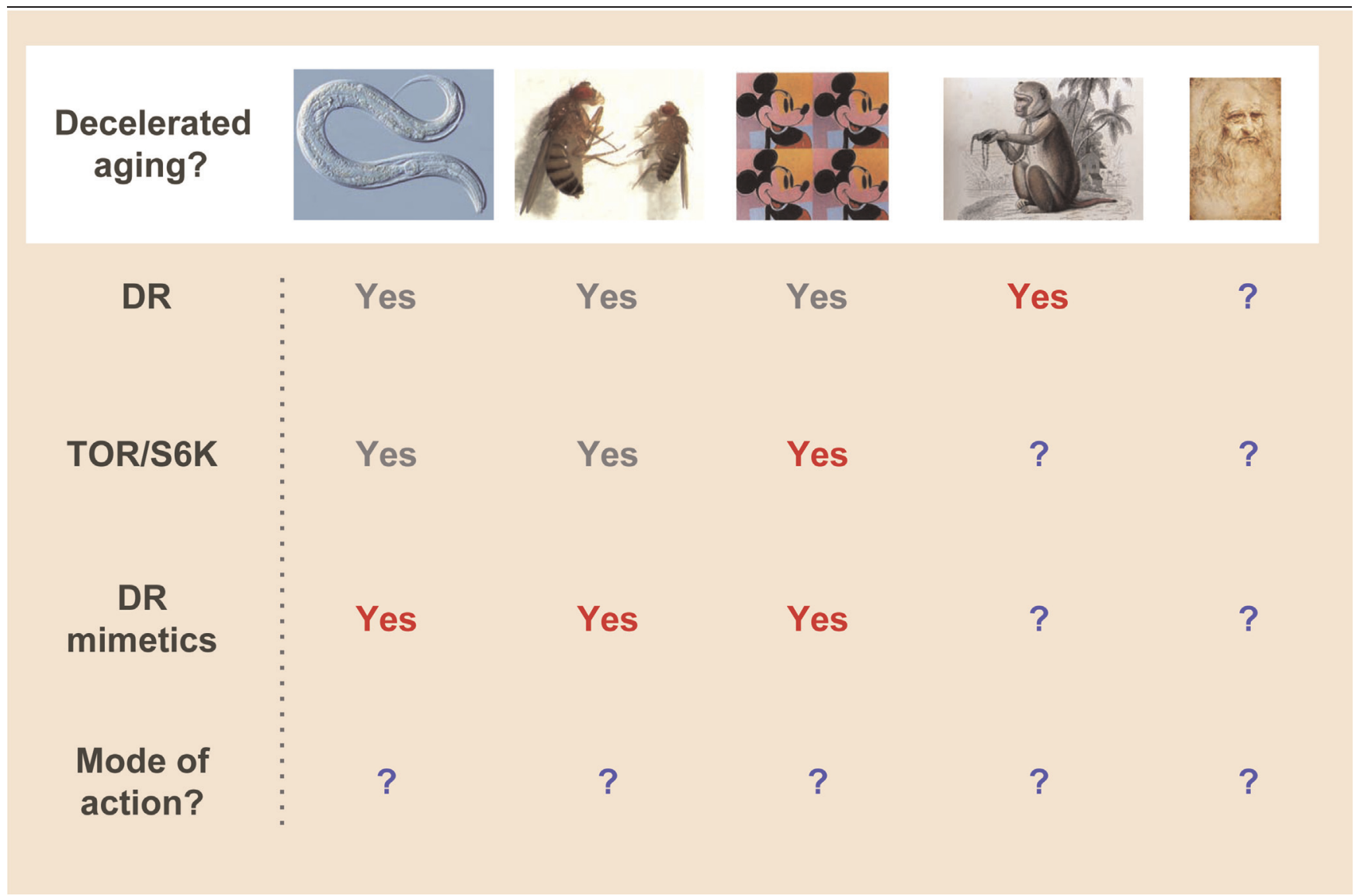

The pathways that mediate the effects of DR have been delineated in model organisms (worms, flies, mice, and rhesus monkeys). The challenges now are to understand how DR works and to apply these findings to humans. Red indicates recent discoveries. S6K, ribosomal S6 kinase; TOR, target of rapamycin. 
difficult to slow in males; for example, effects on aging in S6K1 mutant mice are confined largely to females [10]. The greater phenotypic plasticity (in aging) that females seem to possess warrants further investigation.

Whether DR slows human aging remains unclear. However, studies were initiated in the late 1980s to discover whether DR can increase life span in primates (rhesus macaques). Interim results were reported recently and show that DR does reduce pathology and, potentially, mortality [18], consistent with results of an earlier, smaller trial [19].

\section{Future directions}

These recent discoveries motivate further studies that could pave the way to DR mimetic drugs for humans (Figure 1). Here, many questions remain. For example, does DR slow human aging? There are hints that it might. In Okinawa, Japan, a culture of DR is associated with an elevated frequency of centenarians [20], and in the US, those who practice DR show signs of improved cardiovascular health [21]. But further human studies, such as the ongoing CALERIE (Comprehensive Assessment of the Long-term Effect of Reducing Intake of Energy) study [22], are needed. Also, is there any evidence that metformin, rapamycin, or related compounds might slow human aging? Here, effects on diseases that are aging-related may provide clues. For example, DR reduces cancer incidence, at least in rodents and non-human primates, as do rapamycin and metformin [23]. Another issue is adverse side effects. Metformin can cause gastrointestinal upset, and rapamycin has immunosuppressant effects. To be a practical medical intervention, DR mimetics would need to be effective without inducing significant side effects. A final remaining question is how the TOR/S6K pathway controls aging. To answer this, the puzzle of aging itself will need to be solved.

\section{Abbreviations}

AMPK, adenosine monophosphate-activated kinase; $\mathrm{DR}$, dietary restriction; IIS, insulin/insulin-like growth factor-1 signaling; S6K, ribosomal S6 kinase; TOR, target of rapamycin.

\section{Competing interests}

The authors declare that they have no competing interests.

\section{Acknowledgments}

We thank Ivana Bjedov for reading the manuscript, and we acknowledge funding from a Wellcome Trust Strategic Award and the European Union.

\section{References}

I. Caplan AL: Death as an unnatural process. Why is it wrong to seek a cure for aging? EMBO Rep 2005, 6:S72-5.

2. Gems D: Tragedy and delight: the ethics of decelerated ageing. Phil Trans $R$ Soc, in press.

3. Guarente LP, Partridge L, Wallace DC (eds): Molecular Biology of Aging. Cold Spring Harbor, NY: Cold Spring Harbor Laboratory Press; 2008.

4. Mair W, Dillin A: Aging and survival: the genetics of life span extension by dietary restriction. Annu Rev Biochem 2008, 77:727-54.

5. Ayyadevara S, Alla R, Thaden JJ, Shmookler Reis RJ: Remarkable longevity and stress resistance of nematode PI3K-null mutants. Aging Cell 2008, 7:13-22.

FI000 Factor 3.0 Recommended

Evaluated by David Gems 08 Jan 2008

6. Kenyon C: The genetics of ageing. Nature 2010, 464:504-I2.

7. Ingram DK, Anson RM, de Cabo R, Mamczarz J, Zhu M, Mattison J, Lane MA, Roth GS: Development of calorie restriction mimetics as a prolongevity strategy. Ann N Y Acad Sci 2004, 1019:4|2-23.

8. Schmidt C: GSK/Sirtris compounds dogged by assay artifacts. Nat Biotech 2010, 28:185-6.

9. Steffen KK, MacKay VL, Kerr EO, Tsuchiya M, Hu D, Fox LA, Dang N, Johnston ED, Oakes JA, Tchao BN, Pak DN, Fields S, Kennedy BK, Kaeberlein M: Yeast life span extension by depletion of 60 s ribosomal subunits is mediated by Gcn4. Cell 2008, 133:292-302.

FI000 Factor 6.4 Must Read

Evaluated by Nektarios Tavernarakis 19 May 2008, Thomas Nystrom 28 May 2008

10. Selman C, Tullet JM, Wieser D, Irvine E, Lingard SJ, Choudhury AI, Claret M, Al-Qassab H, Carmignac D, Ramadani F, Woods A, Robinson IC, Schuster E, Batterham RL, Kozma SC, Thomas G, Carling D, Okkenhaug K, Thornton JM, Partridge L, Gems D, Withers DJ: Ribosomal protein S6 kinase I signalling regulates mammalian lifespan. Science 2009, 326:140-4.

FI000 Factor 3.3 Recommended

Evaluated by William H Colledge 12 Oct 2009, Robert Booth 26 Oct 2009, Daniel Gallie 30 Nov 2009

II. Bjedov I, Toivonen JM, Kerr F, Slack C, Jacobson J, Foley A, Partridge $P$ : Mechanisms of lifespan-extension by rapamycin in Drosophila melanogaster. Cell Metab 2010, I I:35-46.

12. Harrison DE, Strong R, Sharp ZD, Nelson JF, Astle CM, Flurkey K, Nadon NL, Wilkinson JE, Frenkel K, Carter CS, Pahor M, Javors MA, Fernandez E, Miller RA: Rapamycin fed late in life extends lifespan in genetically heterogeneous mice. Nature 2009, 460:392-5.

FI000 Factor 8.2 Exceptional

Evaluated by David Gems 03 Aug 2009, Heinz Osiewacz 3 I Jul 2009, David Triggle 20 Aug 2009

13. Kalender A, Selvaraj A, Kim SY, Gulati P, Brûlé S, Viollet B, Kemp BE, Bardeesy N, Dennis P, Schlager JJ, Marette A, Kozma SC, Thomas G: Metformin, independent of AMPK, inhibits $\mathrm{mTORCI}$ in a rag GTPase-dependent manner. Cell Metab 2010, I I:390-40I.

14. Dhahbi JM, Mote PL, Fahy GM, Spindler SR: Identification of potential caloric restriction mimetics by microarray profiling. Physiol Genomics 2005, 23:343-50.

15. Onken B, Driscoll M: Metformin induces a dietary restrictionlike state and the oxidative stress response to extend C. elegans Healthspan via AMPK, LKBI, and SKN-I. PLoS One 2010, 5:e8758.

16. Anisimov VN, Berstein LM, Egormin PA, Piskunova TS, Popovich IG, Zabezhinski MA, Tyndyk ML, Yurova MV, Kovalenko IG, Poroshina TE, Semenchenko AV: Metformin slows down aging and extends life span of female SHR mice. Cell Cycle 2008, 7:2769-73. 
17. Smith DL Jr, Elam CF Jr, Mattison JA, Lane MA, Roth GS, Ingram DK, Allison DB: Metformin supplementation and life span in Fischer-344 rats. J Gerontol A Biol Sci Med Sci 2010, 65:468-74.

18. Colman RJ, Anderson RM, Johnson SC, Kastman EK, Kosmatka KJ, Beasley TM, Allison DB, Cruzen C, Simmons HA, Kemnitz JW, Weindruch $\mathrm{R}$ : Caloric restriction delays disease onset and mortality in rhesus monkeys. Science 2009, 325:20I-4.

FI000 Factor 9.0 Exceptiona

Evaluated by David Gems 29 Jul 2009

19. Bodkin NL, Alexander TM, Ortmeyer HK, Johnson E, Hansen BC: Mortality and morbidity in laboratory-maintained Rhesus monkeys and effects of long-term dietary restriction. J Gerontol A Biol Sci Med Sci 2003, 58:212-9.
20. Kagawa $Y$ : Impact of Westernization on the nutrition of Japanese: changes in physique, cancer, longevity and centenarians. Prev Med 1978, 7:205-17.

21. Fontana L, Meyer T, Klein S, Holloszy J: Long-term calorie restriction is highly effective in reducing the risk for atherosclerosis in humans. Proc Natl Acad Sci U S A 2004, 101:6659-63.

22. Redman LM, Veldhuis JD, Rood J, Smith SR, Williamson D, Ravussin E: The effect of caloric restriction interventions on growth hormone secretion in nonobese men and women. Aging Cell 2010, 9:32-9.

23. Evans JM, Donnelly LA, Emslie-Smith AM, Alessi DR, Morris AD: Metformin and reduced risk of cancer in diabetic patients. BMJ 2005, 330:1304-5. 